\title{
ESCO2 inhibits tumor metastasis via transcriptionally repressing MMP2 in colorectal cancer
}

This article was published in the following Dove Press journal: Cancer Management and Research

\author{
Xiong-Bo Guo ${ }^{1, *}$ \\ Bin Huang 1 ,* \\ Ying-Hua $\mathrm{Pan}^{2}$ \\ Shu-Guang $\mathrm{Su}^{3}$ \\ Yan $\mathrm{Li}^{3}$
}

'Department of General Surgery, Institute of Surgery Research, Daping Hospital, Army Medical University, Chongqing, China; ${ }^{2}$ Department of Rheumatology, The Third Affiliated Hospital of Sun Yat-sen University, Guangzhou, China; ${ }^{3}$ Department of Pathology, The Affiliated Hexian Memorial Hospital of Southern Medical University, Guangzhou, China

*These authors contributed equally to this work
Correspondence: Yan Li

Department of Pathology, The Affiliated Hexian Memorial Hospital of Southern Medical University, 2 Qinghe Road East, Guangzhou, China

Email liyantougao@I63.com
Background: Establishment of cohesion 1 homolog 2 (ESCO2) plays important roles in the regulation of cohesion and genomic stability and has been implicated in human cancers. Yet, its clinical significance and biological function in colorectal cancer (CRC) are unknown.

Methods: The expression of ESCO2 was examined by quantitative real-time PCR, Western blot, and immunohistochemistry. The role of ESCO2 in the tumor metastasis of CRC and the related mechanisms were investigated using in vitro and in vivo models.

Results: In this study, we show that low expression of ESCO2 in CRC was closely correlated with lymphatic and distant metastasis. Patients with low ESCO2 expression experienced shorter overall survival and disease-free survival in two independent cohorts containing a total of $587 \mathrm{CRC}$ cases. ESCO2 overexpression suppressed, whereas ESCO2 knockdown promoted cell migration in vitro and tumor metastasis in vivo via modulation of epithelial-mesenchymal transition (EMT) process. Mechanistically, ESCO2 inhibited the transcriptional activity of MMP2 promoter to downregulate its expression. Reexpression of MMP2 partially attenuated the ESCO2-mediated malignant phenotypes. Conclusion: Collectively, our data suggest that ESCO2 serves as a potential prognostic factor and exerts antimetastatic activity in CRC.

Keywords: ESCO2, MMP2, EMT, metastasis, colorectal cancer

\section{Introduction}

Colorectal cancer (CRC) is the third most prevalent cancer in men and the second in women, and is a leading cause of cancer mortality. ${ }^{1,2}$ The prognosis of CRC patients at early stage is good. However, most of the patients are diagnosed at late stage and are no longer suitable for surgical treatment. It has been well established that approximately $40 \%$ of CRC patients have fatal distant metastasis, mainly to the liver, which makes CRC the leading cause of cancer death. ${ }^{1,3}$ As a result, it is imperative to identify molecular factors that predispose CRC patients to metastatic phenotypes.

Dysregulation of genes involved in spindle assembly and chromosome alignment contributes to the initiation and progression of human diseases. ${ }^{4,5}$ Establishment of cohesion 1 homolog 2 (ESCO2), which was first identified in budding yeast, is an evolutionary conserved cohesion acetyltransferase that exerts essential functions in the establishment of sister chromatid cohesion during S phase and proper chromosome segregation during mitosis. ${ }^{6-9}$ ESCO2 protein consists of a divergent $\mathrm{N}$-terminus followed by a $\mathrm{C} 2 \mathrm{H} 2$ zinc finger and a highly conserved acetyltransferase domain. ${ }^{7}$ Deficiency of ESCO2 leads to abrupt termination of development. ${ }^{10}$ Mutations in human ESCO2 cause a hereditary developmental disease called Roberts syndrome, which is a childhood autosomal recessive disorder that causes mental and physical abnormalities. ${ }^{11,12}$ 
ESCO2 transcriptionally controlled the expression of CX43 through the modification of SMC3 during skeletal regeneration in the zebrafish fin. ${ }^{13}$ It is worth noting that ESCO2 has been linked to cancer progression. ESCO2 is highly expressed in aggressive melanomas and breast cancer. ${ }^{14,15}$ In gastric cancer, ESCO2 promoted cell proliferation by modulating p53 and mammalian target of rapamycin (mTOR) signaling pathways. ${ }^{16}$ However, the biological function and clinical significance of $\mathrm{ESCO} 2$ in $\mathrm{CRC}$ remain unclear.

In this study, the expression of ESCO2 and its clinical implication were determined in two independent cohorts containing a total of 587 patients with $\mathrm{CRC}$. The role of ESCO2 in cell migration, as well as the underlying mechanism, was investigated. Our data indicate that ESCO2 serves as a potential prognostic factor in $\mathrm{CRC}$ and exhibits antimetastatic activity via transcriptional modulation of MMP2.

\section{Materials and methods \\ Clinical samples}

A cohort containing 211 fresh CRC tissue samples and 106 paraffin-embedded tissues was obtained from January 2003 to December 2008 in the Affiliated Hexian Memorial Hospital $(\mathrm{HMH})$ of Southern Medical University (HMH cohort). The mean age of patients was 47.6 years (ranging from 21 to 75 years). The median follow-up was 69 months. Written informed consent was obtained. None of the patients received chemotherapy or radiotherapy before surgery. All samples were anonymous. The use of human samples was approved by the research ethics committee of Southern Medical University and carried out in accordance with the Code of Ethics of the World Medical Association (Declaration of Helsinki). The mRNA expression and prognostic value of ESCO2 were validated in 376 patients with $\mathrm{CRC}$ in The Cancer Genome Atlas (TCGA cohort) through the following website: http:// www.cbioportal.org/.

\section{Cell lines and transfection}

CRC cell lines (DLD1, HCT116, LOVO, and SW620) purchased from the Cell Resource Center, Chinese Academy of Science Committee (Shanghai, China) were maintained in DMEM (Thermo Fisher Scientific, Waltham, MA, USA) supplemented with 10\% heat-inactivated FBS (Hyclone, Logan, UT, USA) in a humidified incubator at $37^{\circ} \mathrm{C}$ and $5 \% \mathrm{CO}_{2}$. The cells were stably transfected with ESCO2 overexpression vector or shRNAs with Lipofectamine 2000. Stable cell lines were established by G418 screening for 2 weeks. The shRNAs for ESCO2 were obtained from Santa Cruz Biotechnology Inc. (Dallas, TX, USA; sc-77558-SH).

\section{Quantitative real-time PCR ( $q R T-P C R$ )}

Complementary DNA was synthesized from the total RNA using the PrimeScript RT reagent Kit (TAKARA, Belmont, NJ, USA). qRT-PCR was performed with SYBR Premix ExTaq (TAKARA). The expression of the endogenous $\beta$-actin was used as control for the normalization of the relative expression of ESCO2. The $-\Delta \mathrm{Ct}$ was calculated. Conditions for RT-PCR were set as follows: $95^{\circ} \mathrm{C}$ for 10 minutes, 40 cycles of $94^{\circ} \mathrm{C}$ for 30 seconds, $60^{\circ} \mathrm{C}$ for 30 seconds, $72^{\circ} \mathrm{C}$ for 30 seconds, and a final extension of 10 minutes at $72^{\circ} \mathrm{C}$. The primers were as follows: ESCO2, forward: 5'-TGGGATAAGTAGAATCTGGGTT-3' and reverse: $5^{\prime}$-ATACGAGGAAATTAGGGGTGT-3'; $\beta$-actin, forward: 5'-TGGCACCCAGCACAATGAA-3' and reverse: 5'-CTAAGTCATAGTCCGCCTAGAAGCA-3'.

\section{Migration and invasion assays}

Cells (for migration assays: $2.0 \times 10^{3}$; for invasion assays: $4.0 \times 10^{3}$ ) were cultured with $200 \mu \mathrm{L}$ serum-free DMEM in the upper compartment of a Transwell chamber (Corning Incorporated, Corning, NY, USA) for 48 hours for the migration assay and 72 hours for the invasion assay. For the invasion assay, the inserts were previously coated with extracellular matrix gel (BD Biosciences, San Jose, CA, USA). The migrated cells were stained with $0.1 \%$ crystal violet and counted under a microscope. The related fold changes of cell migration were shown by histogram.

\section{Luciferase reporter assay}

For the binding of ESCO2 to MMP2 promoter, HCT1 16 cells were cotransfected with ESCO2 overexpression vector and the pRB-REPORT-MMP2 or mutant promoter. Cells were collected 48 hours after transfection, and luciferase activity was analyzed with the Dual Luciferase Reporter Assay System (Promega Corporation, Fitchburg, WI, USA).

\section{Western blot}

Proteins extracted from CRC fresh tissues or cells with various treatments were fractionated by SDS-PAGE, transferred to polyvinylidene difluoride (PVDF) membrane, and then incubated with a primary specific antibody for $\operatorname{ESCO} 2(1: 1,000$, ab86003; Abcam, Cambridge, MA, USA), E-cadherin (1:1,000, sc-14472; Santa Cruz Biotechnology Inc.), N-cadherin (1:1,000, \#13116; Cell Signaling Technology, Danvers, MA, USA), Vimentin (1:1,000, \#5741; Cell Signaling Technology), MMP2 (1:1,000, \#87809; Cell Signaling Technology), active MMP2 (1:500; KeyGen, Jiangsu, China), and $\beta$-actin (1:1,000, \#4970; Cell Signaling Technology) in 5\% of nonfat milk, followed by a horseradish peroxidase (HRP)-conjugated 
anti-rabbit/mouse secondary antibody. Enhanced chemiluminescence (ECL) detection reagent (Amersham Life Science, Piscataway, NJ, USA) was used to visualize the results.

\section{Mice model}

Five-week-old nude BALB/c mice were injected with $5 \times 10^{5}$ cells via the tail vein. Four weeks later, the mice were killed. The lungs of the mice were fixed and stained with H\&E. Lung metastasis was quantified by counting the number of tumor nodules in 10 randomly selected high-power fields. All animal studies were conducted with the approval of the Southern Medical University.

\section{Statistical analyses}

Continuous variables were expressed as mean with SEM and analyzed using the Student's $t$-test (two tailed). Kaplan-Meier analysis (the log-rank test) was used for survival analysis and univariate analysis. The Cox proportional hazards regression model was used to evaluate the independent prognostic value of ESCO2 in CRC. $P$-value of $<0.05$ was considered to be statistically significant.

\section{Results \\ ESCO2 mRNA is upregulated in CRC}

By using transcriptome sequencing (RNA-Seq), we compared the expression of genes involved in cell cycle modulation in eight pairs of CRC and the corresponding nontumorous tissues. We found that ESCO2 expression was significantly higher in CRC tissues than that in normal tissues (Figure 1A). The increase in ESCO 2 mRNA was next validated in an expanded CRC sample cohort containing 211 paired samples (Figure 1B). Among those, 69.2\% of patients had a remarkable ESCO2 upregulation in CRC. Other CRC studies from oncomine database presented that $\mathrm{ESCO} 2$ transcript was upregulated in CRC compared to the nontumorous tissues (Figure 1C). Consistently, the protein level of ESCO2 in $75.0 \%(12 / 16)$ of CRC was much higher than that in normal tissues (Figure 1D). The tissue microarray (TMA)-based immunohistochemistry (IHC) on 106 CRC cases showed that ESCO2 localized in both nuclear and cytoplasm. Positive immunoreactivity was depicted in $81.1 \%(86 / 106)$ of cancerous tissues and $33.0 \%(35 / 106)$ of noncancerous tissues (Figure 1E).

\section{Aberrant expression of ESCO2 was correlated with clinical outcomes in CRC}

The clinical significance of ESCO2 in CRC was next determined in the TCGA cohort. According to the median value of ESCO2 expression, patients were separated into two groups: high ESCO2 and low ESCO2. Interestingly, patients expressing less ESCO2 were frequently accompanied with advanced-stage tumor, tumor metastasis and tumor invasion (Figures $1 \mathrm{~F}$ and S1A and B). This was further confirmed in the HMH cohort. Low ESCO2 expression was significantly associated with poor tumor differentiation $(P=0.021)$, deeper tumor invasion $(P=0.028)$, and lymphatic $(P=0.011)$ and distant tumor metastasis $(P=0.035$; Table 1$)$. We next examined the prognostic value of ESCO2 in CRC. Kaplan-Meier analyses revealed that patients with low ESCO2 expression were frequently accompanied with shorter overall survival and disease-free survival in the HMH cohort (Figure 2A). The prognostic implication of ESCO2 was further validated in the TCGA cohort (Figure 2B). These data indicate that the dysregulation of ESCO2 in CRC may be involved in tumor progression.

\section{ESCO2 exerts antimetastatic activity in CRC}

Since the clinical analyses revealed that ESCO2 expression was closely associated with tumor metastasis, we next examined the biological function of ESCO2 in CRC. ESCO2 was either overexpressed or knocked down in CRC cells (Figure S2). Transwell assays were performed to test the effect of ESCO2 on cell migration and cell invasion. Results showed that ESCO2 overexpression in DLD1 and SW620 cells led to a significant decrease in cell mobility, whereas ESCO2 knockdown in HCT116 and LOVO cells markedly enhanced the capability of cell movement (Figure 3A). The invasive ability of CRC cells was enhanced by the silence of ESCO2, but attenuated by the ectopic expression of ESCO2 (Figure 3B). We next intended to determine whether $\mathrm{ESCO} 2$ was capable of inhibiting tumor metastasis in vivo. After the injection of ESCO2-expressing CRC cells, less lung nodules were developed in the mice, compared to the control group. However, cells with ESCO2 depletion formed much more metastatic tumor in the lungs (Figure $3 \mathrm{C}$ ). These data suggest that ESCO2 exhibits antimetastatic activity in CRC.

\section{ESCO2 transcriptionally represses MMP2 in CRC}

The underlying mechanism of ESCO2-mediated suppression of cell migration was next investigated. Gene set enrichment analysis (GSEA) based on the data from TCGA was performed. Results showed that epithelial-mesenchymal transition (EMT) process was activated in patients with ESCO2 overexpression (Figure S3). As shown in Figure 


\section{A HMH RNA-seq}
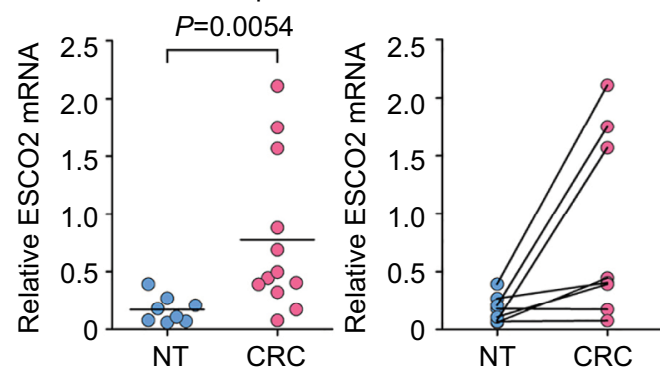

C

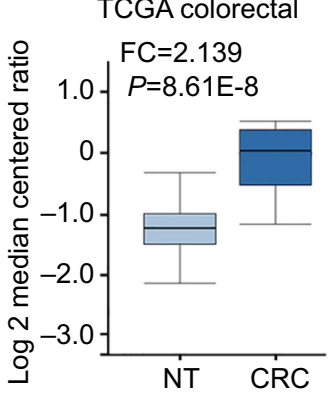

D
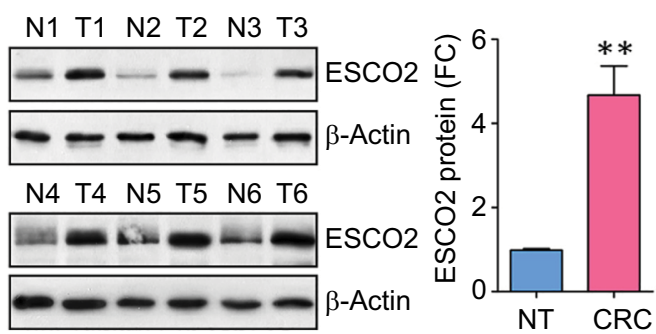

F

Lymph node metastasis

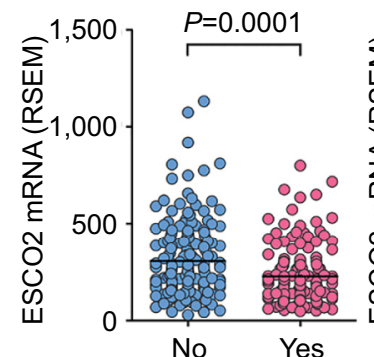

Lymph vascular invasion

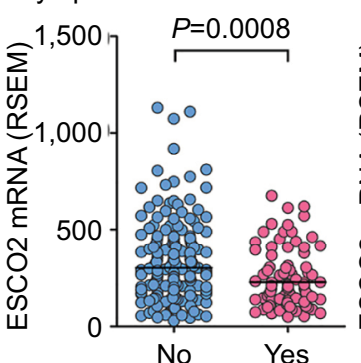

B
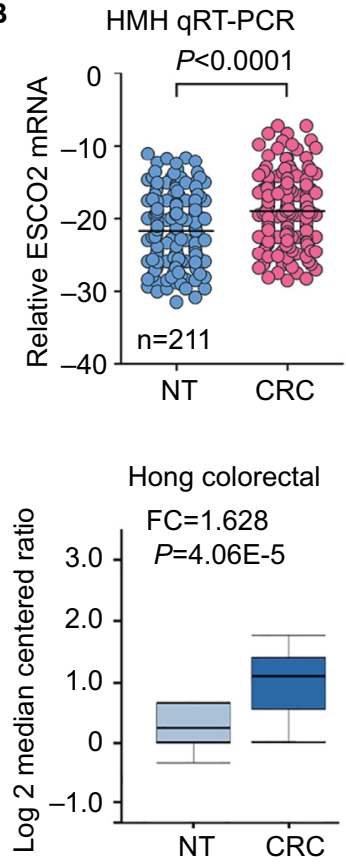

E
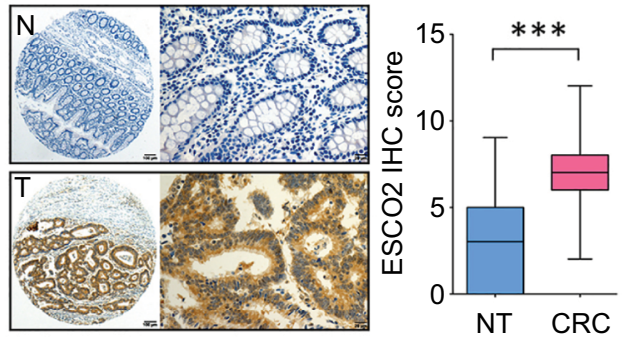

Distal metastasis

Vascular invasion

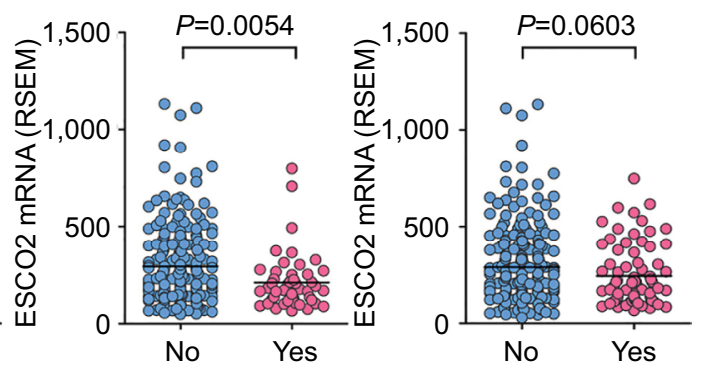

Figure I Schmetic of ESCO2 expression that is increased in CRC.

Notes: (A) Transcriptome sequencing (RNA-Seq) using eight paired fresh CRC and NT tissues and another four CRC specimens was performed. The expression of ESCO2 is indicated (left panel), and the alteration of ESCO2 expression in paired samples is presented (right panel). Horizontal lines: mean expression level. (B) The increase in ESCO2 mRNA was validated in 21 I pairs of clinical specimens using QRT-PCR. (C) ESCO2 upregulation was also found in other studies from oncomine data set. (D) ESCO2 protein expression was determined in 16 paired CRC tissues. Representative Western blot results (left panel) and the related expression levels (right panel) are presented. (E) Expression of ESCO2 protein examined in 106 paraffin-embedded tissues. Representative IHC images (left panel) and scores (right panel) are indicated. (F) Expression of ESCO2 in CRC tissues was compared in patients with or without lymph node metastasis, lymphovascular invasion, distal metastasis, and vascular invasion.

Abbreviations: CRC, colorectal cancer; IHC, immunohistochemistry; NT, nontumorous; qRT-PCR, quantitative real-time PCR; TCGA, The Cancer Genome Atlas; FC, fold change; RSEM, RNA-seq by expectation-maximization.

4A, the ectopic expression of ESCO2 upregulated epithelial marker E-cadherin, but downregulated mesenchymal markers $\mathrm{N}$-cadherin and Vimentin. In contrast, the knockdown of ESCO2 induced the expression of N-cadherin and Vimentin, but reduced the expression of E-cadherin. These data indicate that $\mathrm{ESCO} 2$ participates in EMT process in CRC cells. By using gene microarray, we identified MMP2 as a direct downstream effector of ESCO2 (data not shown). In HCT116 and LOVO cells with ESCO2 silence, the mRNA and protein levels of MMP2 were higher than those in control cells (Figure 4B). Furthermore, the active form of MMP2 was also upregulated by the silence of ESCO2, but down- 
regulated by the ectopic expression of ESCO2 in CRC cells (Figure 4C). Dual luciferase reporter assays demonstrated that an increase in ESCO2 in HCT116 cells significantly inhibited the transcriptional activity of the MMP2 promoter (Figure 4D). In clinical samples, ESCO2 expression was reversely correlated with MMP2 mRNA expression (Figure

Table I Association of ESCO2 expression and clinical features of CRC patients in the HMH cohort

\begin{tabular}{|c|c|c|c|c|}
\hline \multirow[t]{2}{*}{ Variables } & \multirow[t]{2}{*}{$\begin{array}{l}\text { All } \\
\text { cases }\end{array}$} & \multicolumn{2}{|c|}{$\begin{array}{l}\text { ESCO2 mRNA } \\
\text { expression }\end{array}$} & \multirow[t]{2}{*}{$P$-value } \\
\hline & & Low & High & \\
\hline Age (years) & & & & 0.467 \\
\hline$\leq 50$ & 79 & 53 (67.1\%) & $26(32.9 \%)$ & \\
\hline$>50$ & 132 & 82 (62.1\%) & 50 (37.9\%) & \\
\hline Gender & & & & 0.649 \\
\hline Female & 96 & $63(65.6 \%)$ & 33 (34.4\%) & \\
\hline Male & 115 & $72(62.6 \%)$ & 43 (37.4\%) & \\
\hline Size & & & & 0.062 \\
\hline$\leq 4 \mathrm{~cm}$ & 85 & 48 (56.5\%) & 37 (43.5\%) & \\
\hline$>4 \mathrm{~cm}$ & 126 & 87 (69.0\%) & 39 (31.0\%) & \\
\hline Differentiation & & & & 0.021 \\
\hline Well & 4 & $2(50.0 \%)$ & $2(50.0 \%)$ & \\
\hline Moderate & 150 & $88(58.7 \%)$ & $62(41.3 \%)$ & \\
\hline Poor & 57 & 45 (78.9\%) & $12(21.1 \%)$ & \\
\hline $\begin{array}{l}\text { Depth } \\
\text { of tumor } \\
\text { invasion }\end{array}$ & & & & 0.028 \\
\hline $\mathrm{TI}+\mathrm{T} 2$ & 82 & 45 (54.9\%) & 37 (45.1\%) & \\
\hline $\mathrm{T} 3+\mathrm{T} 4$ & 129 & $90(69.8 \%)$ & 39 (30.2\%) & \\
\hline $\begin{array}{l}\text { Lymphatic } \\
\text { metastasis }\end{array}$ & & & & 0.011 \\
\hline Absent & 103 & $55.3 \%$ & $46(44.7 \%)$ & \\
\hline Present & 108 & $72.2 \%$ & 30 (27.8\%) & \\
\hline $\begin{array}{l}\text { Distant } \\
\text { metastasis }\end{array}$ & & & & 0.035 \\
\hline Absent & 154 & $92(59.7 \%)$ & $62(40.3 \%)$ & \\
\hline Present & 57 & 43 (75.4\%) & $14(24.6 \%)$ & \\
\hline
\end{tabular}

Abbreviation: $\mathrm{CRC}$, colorectal cancer.
4E). To determine the role of MMP2 in ESCO2-mediated phenotypes, rescue experiments were performed. Transfection of MMP2 siRNA in cells with ESCO2 depletion markedly attenuated the cell migration (Figure 4F). On the other hand, the suppression of cell migration induced by ESCO2 overexpression was remarkably inhibited by the reexpression of MMP2 (Figure 4F). These findings suggest that ESCO2 exhibits antimetastatic activity via transcriptionally repressing MMP2 in CRC cells.

\section{Discussion}

The high mortality of CRC is mainly attributed to tumor metastasis. ${ }^{3}$ Patients who have been accompanied with recurrent or metastatic disease are no longer suitable for the surgical resection and have to receive conventional cytotoxic chemotherapy and/or targeted therapies. Routine clinical practice including specific molecular marker analyses (eg, microsatellite instability and mutations of RAS and BRAF) may offer references for the clinical management. ${ }^{17}$ Identifying promising metastatic biomarkers and the underlying mechanisms can benefit the therapy of CRC patients. In the present study, we found that $\mathrm{ESCO} 2 \mathrm{mRNA}$ and protein were upregulated. High expression of ESCO2 was correlated with favorable prognosis. Knockdown of ESCO2 expression markedly facilitated cell migration and tumor metastasis. Mechanistic investigations indicated that ESCO2 suppressed EMT process via the downregulation of MMP2. Based on our findings, we raise a hypothesis that $\mathrm{ESCO} 2$ serves as a prognostic factor for $\mathrm{CRC}$ and an antimetastatic factor.

Sister chromatid cohesion is established by Eco1-mediated acetylation on SMC3 at K105 and K106. ${ }^{18}$ Mammalian genomes encode two Eco1 orthologs, ESCO1 and ESCO2. ${ }^{6}$ The two proteins possess distinct expression patterns during cell cycle progression. ${ }^{6} \mathrm{ESCO} 1$ is expressed throughout
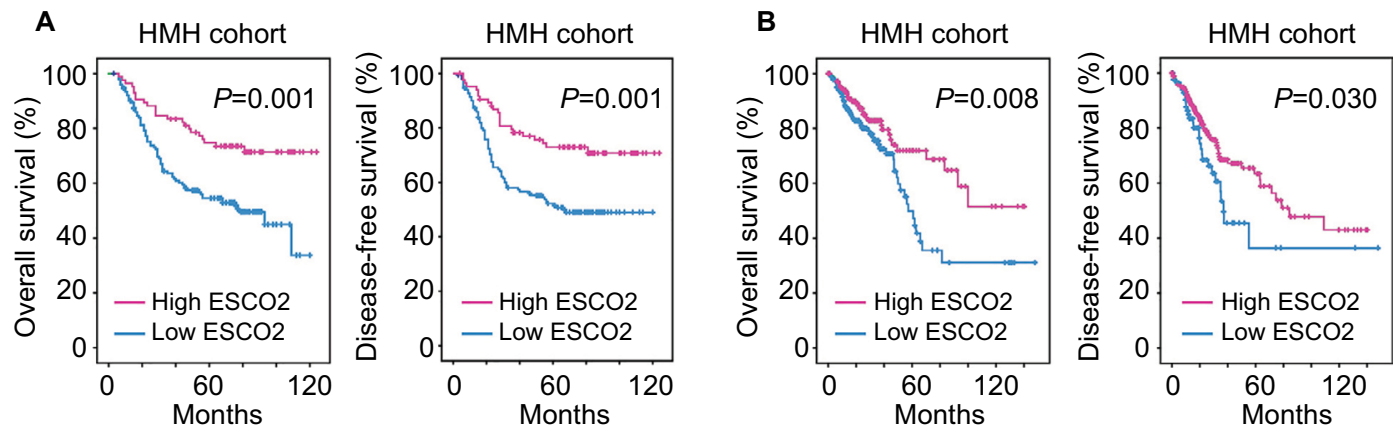

Figure 2 Schmetic of low expression of ESCO2 mRNA that is correlated with poor outcome in CRC.

Notes: (A) The prognostic implication of ESCO2 was revealed by Kaplan-Meier analysis in the HMH cohort. Low expression of ESCO2 mRNA was correlated with unfavorable overall survival (left panel) and disease-free (right panel) survival. (B) The prognostic value of ESCO2 was confirmed in the TCGA cohort.

Abbreviations: CRC, colorectal cancer; TCGA, The Cancer Genome Atlas. 

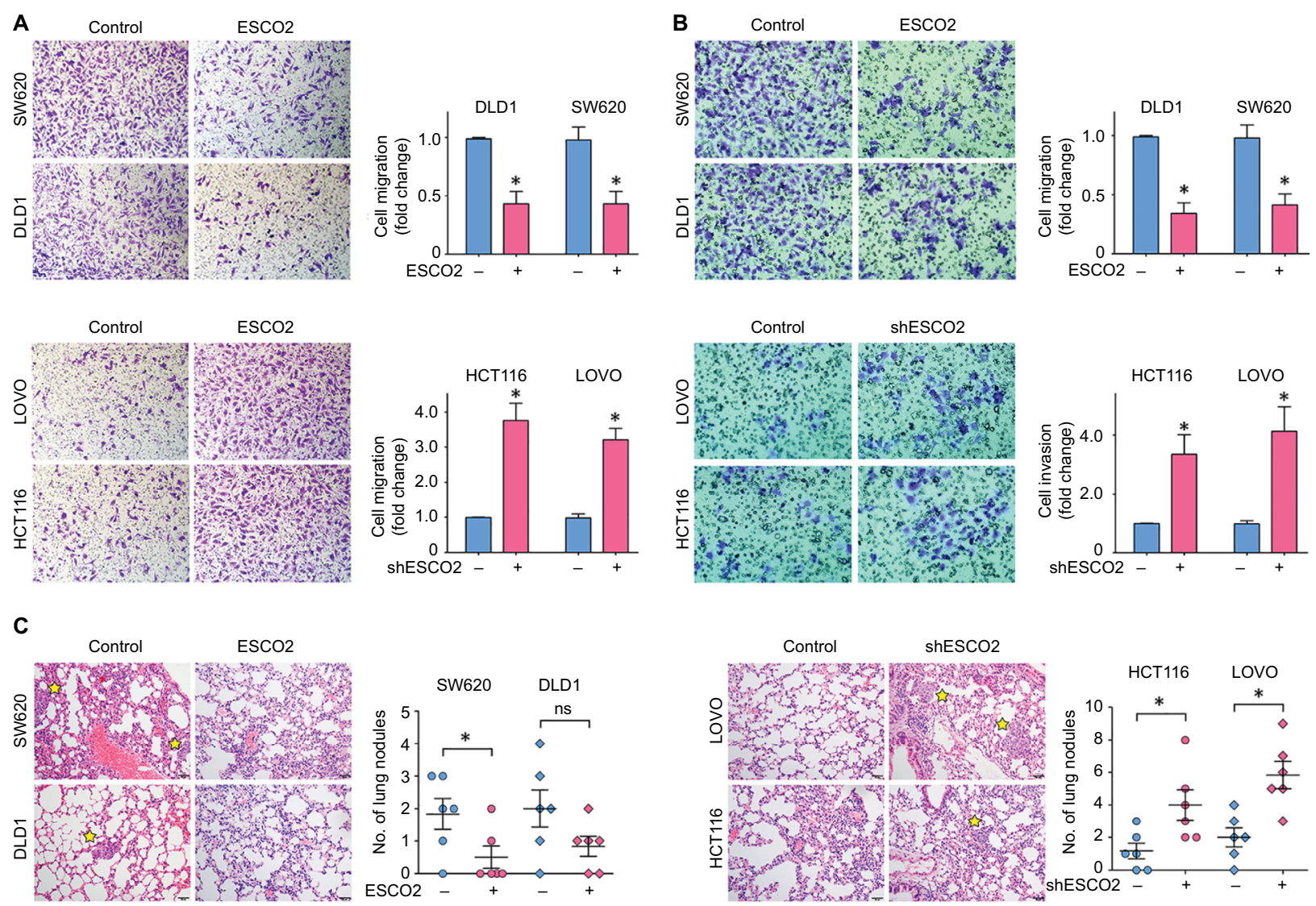

Figure 3 Schmetic of ESCO2 that suppresses cell migration and invasion in vitro and tumor metastasis in vivo.

Notes: (A, B) Cells with ESCO2 overexpression (top panel) and knockdown (bottom panel) were incubated in upper chamber for 48 hours. The migrated cells were stained with $\mathbf{0 . 0 5 \%}$ crystal violet and counted. The representative images of Transwell assays (left panel) and the fold change of cell migration (A) or cell invasion (B) (right panel) are presented. (C) The effect of ESCO2 on tumor metastasis was determined using tail vein injection model. The representative images of lung nodules (left panel) and the number of lung metastases (right panel) in each group are shown. The metastatic nodules in the lungs are indicated by yellow stars. Data are presented as mean $\pm S E M$. $* P<0.05$. Abbreviation: SEM, standard error of the mean.

the cell cycle, while ESCO2 is present mainly in S phase. Dysregulation of ESCO1 has been reported in prostate, ${ }^{19}$ endometrial, ${ }^{20}$ and bladder cancers. ${ }^{21}$ On the other hand, $\mathrm{ESCO} 2$ is overexpressed in melanomas and breast and gastric cancers and is identified as a prognostic factor in breast and lung cancers. ${ }^{15,16,22}$ Yet, its expression and clinical significance in CRC remain unclear. CRC patients bearing large-size tumor but experiencing no tumor metastasis survive longer than those with small-size CRC but accompanied with tumor metastasis, suggesting the notion that metastasis, rather than primary cancer, is the major determinant of the mortality of CRC patients. In our study, ESCO2 expression was increased in CRC tissues, compared with the nontumorous tissues, at both mRNA and protein levels. High expression of ESCO2 was significantly associated with poor tumor differentiation and less tumor metastasis. Furthermore, the elevated expression of ESCO2 was correlated with favorable overall survival and disease-free survival in two independent cohorts consisting of 587 patients with CRC. This was seemingly contradicting. Similar findings were reported in other solid cancers. Transcriptional factor, ZBP-89, was overexpressed and indicated better survival in renal cancer. ${ }^{23}$ High expression of lymphoid enhancer-binding factor-1 (LEF1) was correlated with a novel favorable prognosis in cytogenetically normal acute myeloid leukemia. ${ }^{24} \mathrm{~F}$-box only protein 22 was upregulated in breast cancer and predicted favorable clinical outcomes. ${ }^{25}$ These data indicate that $\mathrm{ESCO} 2$ may restrict CRC in the primary tumor niche.

The current literatures reported that $\mathrm{ESCO} 2$ participated in cell proliferation and differentiation..$^{13,16,26}$ In this study, we provided compelling evidence that ESCO2 was capable of suppressing cell migration in CRC. ESCO2 was suggested as a candidate regulator of meiosis by modulating the transcripts of important genes, such as CX43 or Notch-related genes. ${ }^{13,26}$ On the other hand, ESCO2 exerted functions via protein-protein bindings, such as $\mathrm{SMC} 3$ and $\mathrm{p} 53 .{ }^{16,20}$ In the present study, 
A

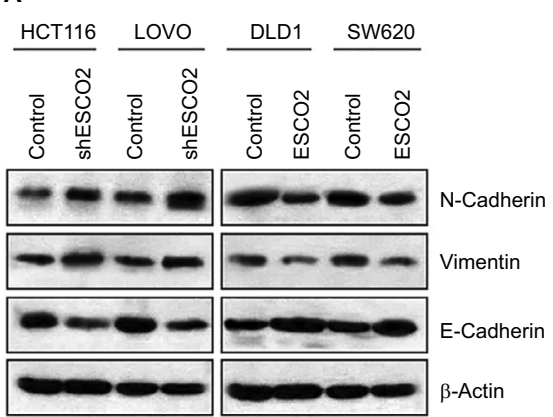

B



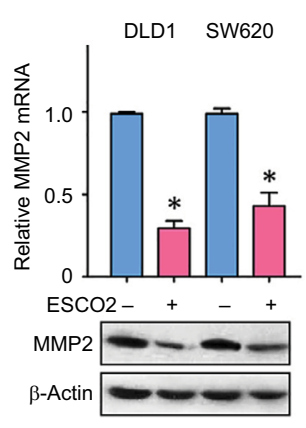

$\mathbf{F}$
C

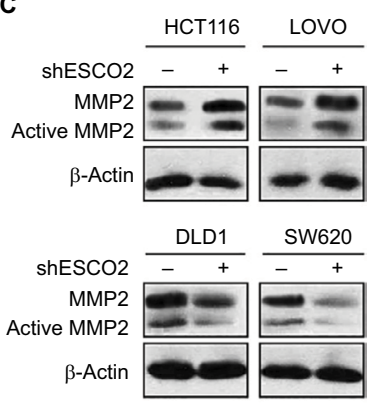

D

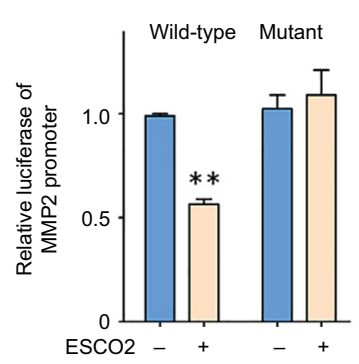

$\mathbf{E}$

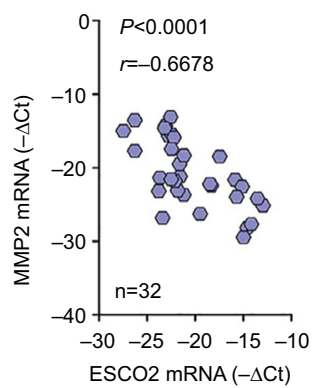

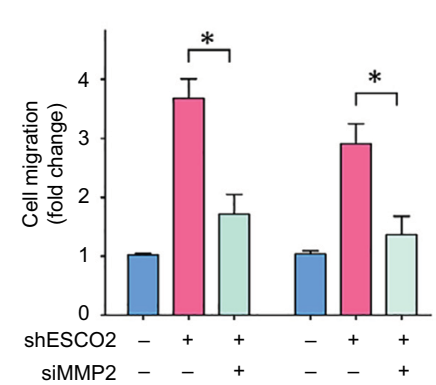

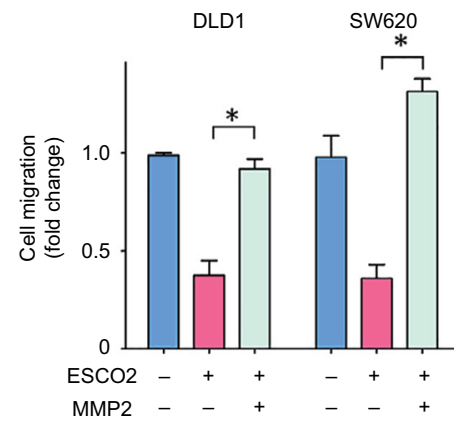

Figure 4 Shemetic of ESCO2 that exerts antimetastatic function by repressing MMP2 expression.

Notes: (A) Proteins extracted from cells with ESCO2 overexpression of depletion were subjected to Western blot. The expressions of EMT markers, N-cadherin, E-cadherin, and Vimentin were examined. (B) The mRNA and protein levels of MMP2 in stable cells were determined using qRT-PCR and Western blot. Data are presented as mean \pm SEM. $* P<0.05$. (C) The active MMP2 expression was determined in CRC cells with altered expression of ESCO2. (D) The effect of ESCO2 overexpression on the activity of MMP2 promoter was measured using luciferase report assays. (E) The mRNA expression of MMP2 and ESCO2 was determined in 32 CRC tissues. The correlation was evaluated. (F) Stable cells were transfected with MMP2 overexpression vector or siRNA for 24 hours. Transwell assays were used to examine the role of MMP2 in ESCO2-mediated cell migration. Data are presented as mean \pm SEM. $* P<0.05$, $* * P<0.01$.

Abbreviations: CRC, colorectal cancer; EMT, epithelial-mesenchymal transition; qRT-PCR, quantitative real-time PCR; SEM, standard error of the mean.

ESCO2 transcriptionally modulated the expression of MMP2, which is involved in the EMT process. ${ }^{27}$ EMT, a dynamic cellular process that is considered as a hallmark of cancer metastasis, involves a decrease in the epithelial marker, such as E-cadherin, and an increase in the levels of mesenchymal markers, such as Vimentin and N-cadherin. ${ }^{28} \mathrm{ESCO} 2$ was found to directly bind to the MMP2 promoter and inhibit its activity, which consequently resulted in a marked downregulation of MMP2 expression and inactivation of MMP2. As a result, the EMT process was blocked and cancer metastasis was suppressed. These data suggest a paradoxical role of ESCO2 in proliferation and metastasis of CRC. Several proteins have been reported to be with pro-proliferative and antimetastatic functions. For example, CBX8 suppressed p53 to enhance tumor growth and inhibited ITGB4 to repress tumor metastasis. ${ }^{29,30}$ FBXO22 played a dual role in tumorigenesis and metastasis via modulation of glycogen synthase kinase $3 \beta$ (GSK3 3 ) and SNAIL. ${ }^{25} \mathrm{c}$-Myc and cyclin A2, two well-established proliferation markers, were able to inhibit cell migration. ${ }^{31,32}$ These data raise the warning that therapeutic strategy based on certain biomarker should be more cautious to avoid the side effect of tumor metastasis. Collectively, our data, for the first time, dem- onstrate that ESCO2 is capable of inhibiting tumor metastasis via the downregulation of MMP2 and therefore suggest ESCO2 as a potential therapeutic target in CRC.

\section{Disclosure}

The authors report no conflicts of interest in this work.

\section{References}

1. Siegel RL, Miller KD, Fedewa SA, et al. Colorectal cancer statistics, 2017. CA Cancer J Clin. 2017;67(3):177-193.

2. Torre LA, Bray F, Siegel RL, Ferlay J, Lortet-Tieulent J, Jemal A. Global cancer statistics, 2012. CA Cancer J Clin. 2015;65(2):87-108.

3. Arnold M, Sierra MS, Laversanne M, Soerjomataram I, Jemal A, Bray F. Global patterns and trends in colorectal cancer incidence and mortality. Gut. 2017;66(4):683-691.

4. Nozawa RS, Boteva L, Soares DC, et al. SAF-A regulates interphase chromosome structure through oligomerization with chromatinassociated RNAs. Cell. 2017;169(7):e1218:1214-1227.

5. Kawasumi R, Abe T, Arakawa H, Garre M, Hirota K, Branzei D. ESCO1/2's roles in chromosome structure and interphase chromatin organization. Genes Dev. 2017;31(21):2136-2150.

6. Alomer RM, da Silva EML, Chen J, et al. Esco1 and Esco2 regulate distinct cohesin functions during cell cycle progression. Proc Natl Acad Sci U S A. 2017;114(37):9906-9911.

7. Kim BJ, Kang KM, Jung SY, et al. Esco2 is a novel corepressor that associates with various chromatin modifying enzymes. Biochem Biophys Res Commun. 2008;372(2):298-304. 
8. Percival SM, Thomas HR, Amsterdam A, et al. Variations in dysfunction of sister chromatid cohesion in esco2 mutant zebrafish reflect the phenotypic diversity of Roberts syndrome. Dis Model Mech. 2015;8(8):941-955.

9. Whelan G, Kreidl E, Peters JM, Eichele G. The non-redundant function of cohesin acetyltransferase Esco2: some answers and new questions. Nucleus. 2012;3(4):330-334.

10. Resta N, Susca FC, Di Giacomo MC, et al. A homozygous frameshift mutation in the ESCO2 gene: evidence of intertissue and interindividual variation in Nmd efficiency. $J$ Cell Physiol. 2006;209(1):67-73.

11. Banerji R, Skibbens RV, Iovine MK. Cohesin mediates Esco2-dependent transcriptional regulation in a zebrafish regenerating fin model of Roberts Syndrome. Biol Open. 2017;6(12):1802-1813.

12. Vega H, Waisfisz Q, Gordillo M, et al. Roberts syndrome is caused by mutations in ESCO2, a human homolog of yeast ECO1 that is essential for the establishment of sister chromatid cohesion. Nat Genet. 2005;37(5):468-470.

13. Banerji R, Eble DM, Iovine MK, Skibbens RV. Esco2 regulates cx43 expression during skeletal regeneration in the zebrafish fin. Dev Dyn. 2016;245(1):7-21.

14. Ryu B, Kim DS, Deluca AM, Alani RM. Comprehensive expression profiling of tumor cell lines identifies molecular signatures of melanoma progression. PLoS One. 2007;2(7):e594.

15. Xiao B, Chen L, Ke Y, et al. Identification of methylation sites and signature genes with prognostic value for luminal breast cancer. $B M C$ Cancer. 2018;18(1):405.

16. Chen H, Zhang L, He W, et al. ESCO2 knockdown inhibits cell proliferation and induces apoptosis in human gastric cancer cells. Biochem Biophys Res Commun. 2018;496(2):475-481.

17. Salvatore L, Aprile G, Arnoldi E, et al. Management of metastatic colorectal cancer patients: guidelines of the Italian Medical Oncology Association (AIOM). ESMO Open. 2017;2(1):e000147.

18. Minamino M, Ishibashi M, Nakato R, et al. Esco1 acetylates cohesin via a mechanism different from that of Esco2. Curr Biol. 2015;25(13):1694-1706.

19. Luedeke M, Linnert CM, Hofer MD, et al. Predisposition for TMPRSS2ERG fusion in prostate cancer by variants in DNA repair genes. Cancer Epidemiol Biomarkers Prev. 2009;18(11):3030-3035.
20. Rivera-Colón Y, Maguire A, Liszczak GP, Olia AS, Marmorstein R. Molecular basis for cohesin acetylation by establishment of sister chromatid cohesion N-acetyltransferase ESCO1. J Biol Chem. 2016;291(51):26468-26477.

21. Zhang S, Li J, Zhou G, et al. Increased expression of ESCO1 is correlated with poor patient survival and its role in human bladder cancer. Tumour Biol. 2016;37(4):5165-5170.

22. Zhang W, Cui Q, Qu W, Ding X, Jiang D, Liu H. TRIM58/cg26157385 methylation is associated with eight prognostic genes in lung squamous cell carcinoma. Oncol Rep. 2018;40(1):206-216.

23. Cai MY, Luo RZ, Li YH, et al. High-expression of ZBP-89 correlates with distal metastasis and poor prognosis of patients in clear cell renal cell carcinoma. Biochem Biophys Res Commun. 2012;426(4):636-642.

24. Metzeler KH, Heilmeier B, Edmaier KE, et al. High expression of lymphoid enhancer-binding factor-1 (LEF1) is a novel favorable prognostic factor in cytogenetically normal acute myeloid leukemia. Blood. 2012;120(10):2118-2126.

25. Sun R, Xie HY, Qian JX, et al. FBXO22 possesses both protumorigenic and antimetastatic roles in breast cancer progression. Cancer Res. 2018;78(18):5274-5286.

26. Leem YE, Choi HK, Jung SY, et al. Esco2 promotes neuronal differentiation by repressing Notch signaling. Cell Signal. 2011;23(11):1876-1884.

27. Xu W, Xu H, Fang M, Wu X, Xu Y. MKL1 links epigenetic activation of MMP2 to ovarian cancer cell migration and invasion. Biochem Biophys Res Commun. 2017;487(3):500-508.

28. Marcucci F, Stassi G, De Maria R. Epithelial-mesenchymal transition: a new target in anticancer drug discovery. Nat Rev Drug Discov. 2016;15(5):311-325.

29. Tang J, Wang G, Zhang M, et al. Paradoxical role of CBX8 in proliferation and metastasis of colorectal cancer. Oncotarget. 2014;5(21):10778-10790.

30. Zhang $\mathrm{CZ}$, Chen $\mathrm{SL}$, Wang $\mathrm{CH}$, et al. CBX8 exhibits oncogenic activity via $\mathrm{AKT} / \beta$-catenin activation in hepatocellular carcinoma. Cancer Res. 2018;78(1):51-63.

31. Liu H, Radisky DC, Yang D, et al. MYC suppresses cancer metastasis by direct transcriptional silencing of $\alpha \mathrm{v}$ and $\beta 3$ integrin subunits. Nat Cell Biol. 2012;14(6):567-574.

32. Arsic N, Bendris N, Peter M, et al. A novel function for Cyclin A2: control of cell invasion via RhoA signaling. J Cell Biol. 2012;196(1):147-162. 


\section{Supplementary materials}

A

Lymph node metastasis

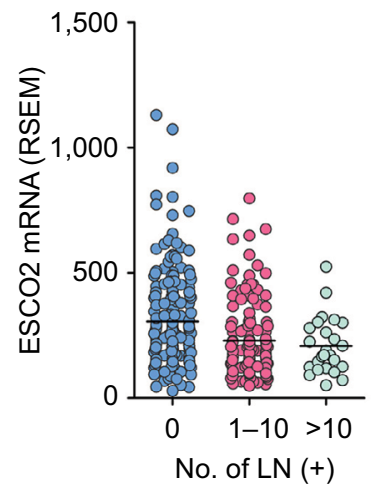

B

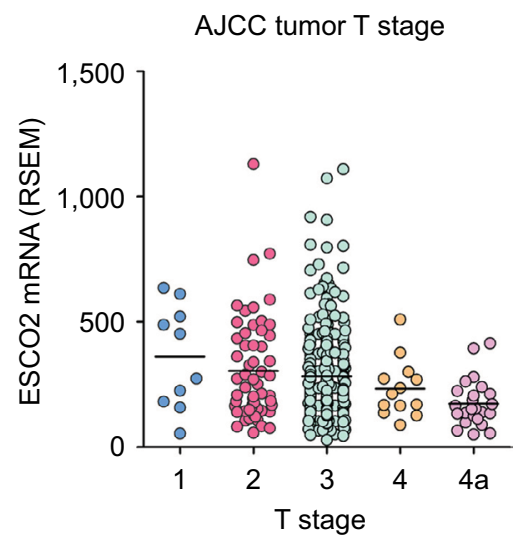

Figure SI Association of ESCO2 mRNA expression and clinical features such as lymph node metastasis and T stage.

Notes: (A) ESCO2 mRNA expression in patients with or without lymph node metastasis. (B) ESCO2 mRNA expression in patients at different T stage. Abbreviations: RSEM, RNA-seq by expectation-maximization; LN, lymph node; AJCC, American Joint Committee on Cancer.

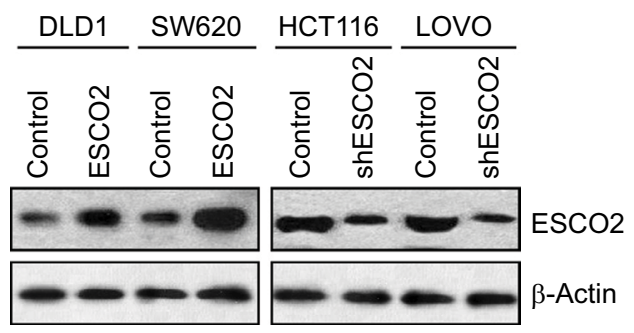

Figure S2 Western blot analysis to show the overexpression or silence of ESCO2 protein in CRC cell lines.

Abbreviation: CRC, colorectal cancer.

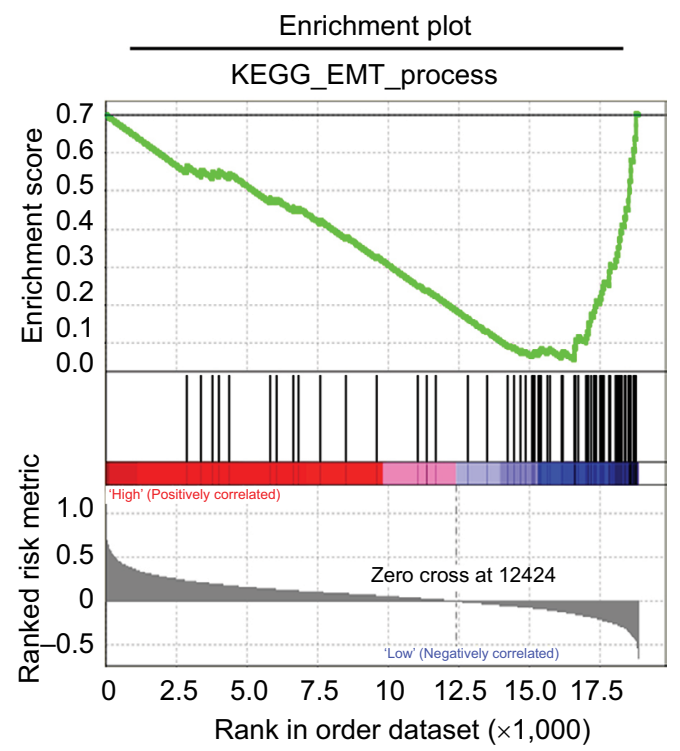

Figure S3 GSEA based on the data from TCGA.

Abbreviations: EMT, epithelial-mesenchymal transition; GSEA, gene set enrichment analysis; TCGA, The Cancer Genome Atlas; KEGG, Kyoto Encyclopedia of Genes and Genomes. 


\section{Publish your work in this journal}

Cancer Management and Research is an international, peer-reviewed open access journal focusing on cancer research and the optimal use of preventative and integrated treatment interventions to achieve improved outcomes, enhanced survival and quality of life for the cancer patient. The manuscript management system is completely online and includes a very quick and fair peer-review system, which is all easy to use. Visit http://www.dovepress.com/testimonials.php to read real quotes from published authors.

Submit your manuscript here: https://www.dovepress.com/cancer-management-and-research-journal 